\title{
Evaluation of sorption properties for cesium on illite-rich clay using cation exchange model
}

JEONGHWAN HWANG ${ }^{1}$,SUNGWOOK CHOUNG ${ }^{2}$, JAEHYUN LEE $^{1}$, WEON SHIK HAN ${ }^{1, *}$

${ }^{1}$ Yonsei University, 50 Yonsei-Ro, Seodaemun-Gu, Seoul 03722, Republic of Korea

${ }^{2}$ Korea Basic Science Institute (KBSI), 162 Yeongudanji-Ro, Cheongju 28119, Republic of Korea

Clay mineral is an important barrier material in the high-level waste disposal repository because its high sorption capacities for radionuclides. However, it is necessary to evaluate the sorption characteristics of clay material precisely due to their heterogeneous properties. The cation exchange model (CEM) is one of the powerful modelling methods for evaluating the sorption characteristics of clay minerals. Major assumption of CEM is that the permanent negative charges are distributed on three exchange sites, each having different site capacities and sorption affinities, of clay mineral surfaces. These sites (i.e., frayed edge sites, type II sites [high affinity and low capacity] and planar sites [low affinity but high capacity]) are coupled to each other to form a complex sorption mechanism.

In this study, we used total 3 illite-rich clay samples (IC1 to IC3), collected from Yeongdong, South Korea, to evaluate the sorption property for Cs utilizing CEM. All samples were identified with major illite components ( $\approx 70 \%)$ using XRD analysis, and their cation exchange capacities were determined to be about $0.05-0.09 \mathrm{eq} \mathrm{kg}^{-1}$. Batch sorption experiments were performed at $10^{-7}-10^{-3} \mathrm{M}$ of Cs concentration using 3 types of background solutions (DIW, $10^{-3} \mathrm{M}, 10^{-1} \mathrm{M} \mathrm{KCl}$ ), and the CEM was developed using geochemical program PHREEQC v.3 (USGS). The sorption capabilites of ICs derived from experiments significantly decreased with an increase of cation concentration, majorly K, at background solution. The CEM accurately evaluated sorption characteristics of exchangable sites, especially frayed edge sites and planar sites at experimental Cs range. The further study is coupling CEM with surface complexation model (SCM) to improve the model and appling to 1D transport model to evaluate the migration of Cs, precisely. 